\title{
Semi-discrete isothermic surfaces
}

\author{
F. Burstall ${ }^{1}$ (D) U. Hertrich-Jeromin ${ }^{2}$. \\ C. Müller ${ }^{2} \cdot$ W. Rossman ${ }^{3}$
}

Received: 26 August 2015 / Accepted: 22 January 2016 / Published online: 13 February 2016

(C) The Author(s) 2016. This article is published with open access at Springerlink.com

\begin{abstract}
A Darboux transformation for polarized space curves is introduced and its properties are studied, in particular, Bianchi permutability. Semi-discrete isothermic surfaces are described as sequences of Darboux transforms of polarized curves in the conformal $n$-sphere and their transformation theory is studied. Semi-discrete surfaces of constant mean curvature are studied as an application of the transformation theory.
\end{abstract}

Keywords Isothermic surface - Discrete isothermic net - Calapso transformation - Darboux transformation - Bianchi permutability · Lawson correspondence · Bäcklund transformation · Constant mean curvature

Mathematics Subject Classification 2010 53A10 - 53C42 · 53A30 · 37K25 - 37K35

\section{Introduction}

Integrable discretizations of surfaces or submanifolds are intimately related to the transformations of the smooth theory-permutability theorems govern consistency of the discretization in this setting. This line of thought has probably been most clearly formulated in [4] or [5]:

\footnotetext{
$\bowtie \quad$ F. Burstall

feb@maths.bath.ac.uk

U. Hertrich-Jeromin

udo.hertrich-jeromin@tuwien.ac.at

C. Müller

cmueller@geometrie.tuwien.ac.at

W. Rossman

wayne@math.kobe-u.ac.jp

1 Department of Mathematical Sciences, University of Bath, Bath BA2 7AY, UK

2 Vienna University of Technology, Wiedner Hauptstraße 8-10/104, 1040 Vienna, Austria

3 Department of Mathematics, Kobe University, Rokko, Kobe 657-8501, Japan
} 
"In this setting, discrete surfaces appear as two-dimensional layers of multidimensional discrete nets, and their transformations correspond to shifts in the transversal lattice directions. A characteristic feature of the theory is that all lattice directions are on equal footing with respect to the defining geometric properties."

For example, given two Darboux transforms of an isothermic surface, a fourth surface can be constructed by algebraic means so that the four surfaces form a quadrilateral of surfaces with Darboux transforms along the edges of the quadrilateral; moreover, the (spectral) parameters of the transformations on opposite edges are equal. Thus, repeated transformations and application of this permutability theorem generate a lattice of isothermic surfacestrailing the effect on a single point then generates a 2-dimensional grid in space: a discrete surface with similar properties as a smooth isothermic surface. In particular, the discrete surfaces obtained in this way admit very similar transformations to their smooth analogues, hence generating multi-dimensional lattices that display the same properties in all lattice directions, cf [4]: "Discrete surfaces and their transformations become indistinguishable."

Our principal aim here is to explore this relation between analogous smooth and discrete theories in a setting where the interplay between them becomes most tangible, that is, we unify the smooth and discrete aspects in a single geometric object: a semi-discrete isothermic surface.

Thus, to construct the semi-discrete isothermic surfaces considered in this text, we follow the idea outlined above, see also [13]: we obtain a notion of Darboux transformation for space curves by observing how the Darboux transformation of an isothermic surface acts on a single curvature line, cf [18]—-then we construct semi-discrete isothermic surfaces as sequences of Darboux transforms of curves.

The first part of the text is concerned with the analysis of the Darboux transformation of curves in Euclidean and conformal $n$-space: we introduce the Darboux transformation as a special type of Ribaucour transformation, cf [3] and [16, Sect 8.2], that preserves a quadratic differential as an additional structure on a curve - this Darboux transformation acts on curves equipped with a quadratic differential, reminiscent of the fact that the Darboux transformation of an isothermic surface preserves a holomorphic quadratic differential that is associated to every isothermic surface. This notion of Darboux transformation for polarized curves generalizes the notions of [18, Sect 2.6].

In particular, Darboux transformations are given by solutions of a Riccati equation that includes a (spectral) parameter-we discuss a linearization of this Riccati equation using Möbius geometric techniques, by means of a connection on a suitable vector bundle, reflecting the conformal invariance of the transformation. Darboux transforms are then obtained from parallel sections of this connection, that is, as solutions of Darboux's linear system. A version of the aforementioned Bianchi permutability theorem for the Darboux transformation of curves is then derived using standard gauge theoretic arguments.

Semi-discrete isothermic surfaces are introduced in the second part of the text, as sequences of Darboux transforms of curves. As detailed above in the fully discrete case, the Darboux transformation for semi-discrete isothermic surfaces can then be consistently defined because of the corresponding Bianchi permutability theorem for the Darboux transformation of curves. Further, we discuss how the Christoffel and Calapso transformations of semidiscrete isothermic surfaces occur from corresponding transformations for curves and suitable permutability theorems.

In summary, we do not only obtain the full transformation theory for semi-discrete isothermic surfaces, but, relying on the aforementioned linearization of the Riccati equation that describes the Darboux transformation, we also obtain a gauge theoretic characterization of semi-discrete isothermic surfaces, as are known in the smooth and fully discrete cases, cf [9]. 
As an example of the developed transformation theory and gauge theoretic approach for semi-discrete isothermic surfaces, we discuss semi-discrete surfaces of constant mean curvature in space forms, thereby extending results from $[19,20]$. Here we rely on the intimate relation between the Christoffel transformation and a mixed area that is used to define the mean curvature of a surface with Gauss map in a space form, $\mathrm{cf}[6,10]$ for the discrete case; a characterization in terms of linear conserved quantities, $\operatorname{cf}[8,11]$, is then a direct consequence of the gauge theoretic characterization of semi-discrete isothermic surfaces and its relation with the Christoffel transformation.

\section{Darboux transformations of curves}

Our idea is that semi-discrete isothermic surfaces should be obtained by restricting a sequence of Darboux transforms of an isothermic surface to a fixed curvature line. We therefore seek a notion of "Darboux transformation" for (decorated) curves in space and shall find it in much the same way that a "Bäcklund transformation" for curves of constant torsion was found by restricting Bäcklund transformations of pseudo-spherical surfaces to a single asymptotic line in [13, Sect 1.1]. In particular, the Darboux transformation will occur as a special type of Ribaucour transformation, cf [16, Sect 8.2] or [3, Sect 3]: the Ribaucour transformation of hypersurfaces or submanifolds descends to their curvature lines, where only the enveloping condition remains a non-trivial condition.

Definition 2.1 Two curves $x, \hat{x}: I \rightarrow \mathbb{R}^{n}$ will be said to form a Ribaucour pair if they envelop a circle congruence, i.e., if tangents at corresponding points $x(s)$ and $\hat{x}(s)$ are tangent to a common circle $c(s)$. Either curve of a Ribaucour pair is a Ribaucour transform of the other curve.

Using a semi-discrete version of the Clifford algebra cross ratio of [14], cf [7, Sect 2.4], we obtain an algebraic characterization of Ribaucour pairs of curves:

Definition and Lemma 2.2 A pair of curves $(x, \hat{x}): I \rightarrow \mathbb{R}^{n} \times \mathbb{R}^{n}$ is a Ribaucour pair if and only if its tangent cross ratio

$$
c r:=x^{\prime}(x-\hat{x})^{-1} \hat{x}^{\prime}(x-\hat{x})^{-1}: I \rightarrow \mathbb{R}
$$

is real, where Clifford multiplication is used for products and inverses. In that case, the tangent cross ratio is symmetric,

$$
x^{\prime}(x-\hat{x})^{-1} \hat{x}^{\prime}(x-\hat{x})^{-1}=\hat{x}^{\prime}(\hat{x}-x)^{-1} x^{\prime}(\hat{x}-x)^{-1} .
$$

Namely, $(x, \hat{x})$ envelop a circle congruence if and only if reflection in the bisector of the secants exchanges the tangents, that is, if and only if

$$
x^{\prime} \|(\hat{x}-x)^{-1} \hat{x}^{\prime}(\hat{x}-x)=x^{\prime} \frac{(\hat{x}-x)^{2} c r}{x^{\prime 2}} .
$$

Note that corresponding tangents of a Ribaucour pair of curves generically intersect in a point $y$, which provides an alternative method to determine the tangent cross ratio of a Ribaucour pair from a suitable ratio of scaling factors:

$$
\hat{x}+\frac{1}{\hat{r}} \hat{x}^{\prime}=y=x+\frac{1}{r} x^{\prime}, \text { where } \frac{\hat{r}}{r}=-\frac{(\hat{x}-x)^{2} c r}{x^{\prime 2}}
$$


since

$$
\hat{x}^{\prime}+\frac{(\hat{x}-x)^{2} c r}{x^{\prime 2}} x^{\prime}=\hat{x}^{\prime}+(\hat{x}-x)^{-1} \hat{x}^{\prime}(\hat{x}-x) \|(\hat{x}-x) .
$$

On the other hand, given a tangent cross ratio function $\mathrm{cr}: I \rightarrow \mathbb{R}$, a corresponding Ribaucour transform $\hat{x}$ of a curve $x: I \rightarrow \mathbb{R}^{n}$ can be recovered as a solution of the Riccati equation

$$
\hat{x}^{\prime}=\operatorname{cr}(\hat{x}-x)\left(x^{\prime}\right)^{-1}(\hat{x}-x) .
$$

Our next mission will be to linearize this equation by means of standard Möbius geometric methods, see for example [16, Chap 1]: we will replace the Euclidean $\mathbb{R}^{n}$ as an ambient space by the conformal $n$-sphere $S^{n}$, thought of as the projective light cone of the $(n+2)$ dimensional Minkowski space $\mathbb{R}^{n+1,1}$,

$$
S^{n} \cong \mathbb{P}\left(\mathcal{L}^{n+1}\right), \text { where } \mathcal{L}^{n+1}=\left\{y \in \mathbb{R}^{n+1,1} \mid(y, y)=0\right\} .
$$

Thus a (smooth) choice of homogeneous coordinates for a curve in the conformal $n$-sphere $S^{n}$ constitutes a lift of the curve into the light cone $\mathcal{L}^{n+1}$. In particular, for lightlike vectors $o, q \in \mathbb{R}^{n+1,1}$ with inner product $(o, q)=-1$ consider the orthogonal decomposition

$$
\mathbb{R}^{n+1,1}=\mathbb{R}^{1,1} \oplus_{\perp} \mathbb{R}^{n}=\langle o, q\rangle \oplus_{\perp}\langle o, q\rangle^{\perp},
$$

where $\langle\ldots\rangle$ denotes the span of vectors. Then the Euclidean lift of an immersed curve (or, more generally, submanifold) in $\mathbb{R}^{n}$ is obtained from the isometry

$$
\mathbb{R}^{n} \ni x \mapsto \xi:=o+x+\frac{1}{2}(x, x) q \in Q^{n}:=\left\{y \in \mathcal{L}^{n+1} \mid(y, q)=-1\right\}
$$

into the light cone, realizing the Euclidean geometry of $\mathbb{R}^{n}$ as a subgeometry of the conformal geometry of $S^{n}$, cf [16, Sect 1.4]. Hence identifying $\mathbb{R}^{n} \cong Q^{n}$ as a subset of the conformal $n$-sphere $S^{n}$ by means of (2.4), we will also refer to any light cone map

$$
\xi: I \rightarrow \mathcal{L}^{n+1} \text { with }\langle\xi\rangle=\left\langle o+x+\frac{1}{2}(x, x) q\right\rangle
$$

as a lift of a curve $x: I \rightarrow \mathbb{R}^{n} \subset \mathbb{R}^{n+1,1}$ in Euclidean space. Further, identifying $\Lambda^{2} \mathbb{R}^{n+1,1} \cong$ $\mathfrak{o}\left(\mathbb{R}^{n+1,1}\right)$ via

$$
\xi \wedge \eta: \mathbb{R}^{n+1,1} \ni y \mapsto(\xi \wedge \eta)(y):=(y, \xi) \eta-(y, \eta) \xi \in \mathbb{R}^{n+1,1},
$$

we linearize (2.3) by means of a connection $\frac{D}{d s}$ along $x: I \rightarrow \mathbb{R}^{n} \subset \mathbb{R}^{n+1,1}$, associated to a given tangent cross ratio function $\mathrm{cr}: I \rightarrow \mathbb{R}$ :

Lemma 2.3 A curve $\hat{x}: I \rightarrow \mathbb{R}^{n}$ is a Ribaucour transform of a curve $x: I \rightarrow \mathbb{R}^{n}$ with tangent cross ratio function $\mathrm{cr}: I \rightarrow \mathbb{R}$ if and only if, for any lifts $\xi$ and $\hat{\xi}$ of $x$ and $\hat{x}$, respectively,

$$
\frac{D}{d s} \hat{\xi} \| \hat{\xi}, \text { where } \frac{D}{d s}:=\frac{d}{d s}-2 c r \frac{\xi \wedge \xi^{\prime}}{\left(\xi^{\prime}, \xi^{\prime}\right)}
$$

Using Euclidean lifts $\xi$ and $\hat{\xi}$ of two curves $x$ resp $\hat{x}$ in $\mathbb{R}^{n},(2.5)$ reads

$$
\lambda \hat{\xi}+\hat{\xi}^{\prime}=\frac{2 c r}{\left(\xi^{\prime}, \xi^{\prime}\right)}\left((\hat{\xi}, \xi) \xi^{\prime}-\left(\hat{\xi}, \xi^{\prime}\right) \xi\right)=\frac{2 c r\left(x-\hat{x}, x^{\prime}\right)}{\left(x^{\prime}, x^{\prime}\right)} \xi-\frac{(\hat{x}-x)^{2} c r}{x^{\prime 2}} \xi^{\prime},
$$


where $\lambda=\frac{2 c r\left(x-\hat{x}, x^{\prime}\right)}{\left(x^{\prime}, x^{\prime}\right)}$ by taking inner product with $q$; hence comparing $\mathbb{R}^{n}$-parts of the equation we recover (2.2), which yields the claim. Conversely, assuming (2.2), expansion of

$$
0=\left(\hat{x}-x, y-\frac{\hat{x}+x}{2}\right) \text { yields } \frac{D}{d s} \hat{\xi}=\frac{2 c r\left(x-\hat{x}, x^{\prime}\right)}{\left(x^{\prime}, x^{\prime}\right)} \hat{\xi} .
$$

Moreover, (2.5) is independent of the choice of lifts $\xi$ resp $\hat{\xi}$ : multiplication of $\xi$ by a non-zero function does not change $\frac{D}{d s}$ since $(\xi, \xi) \equiv 0$ and multiplication of $\hat{\xi}$ by a non-zero function does obviously not affect (2.5) as $\frac{D}{d s}$ satisfies Leibniz' rule. This reflects the fact that the Ribaucour transformation is well defined for curves in the conformal $S^{n} \cong \mathbb{P}\left(\mathcal{L}^{n+1}\right)$ : on a 1-dimensional domain the only non-trivial condition for the Ribaucour transformation is the enveloping condition, that is, the condition that

$$
\left\langle\xi, \xi^{\prime}, \hat{\xi}, \hat{\xi}^{\prime}\right\rangle \cong \mathbb{R}^{2,1} \subset \mathbb{R}^{n+1,1}
$$

define a circle congruence, $\operatorname{cf}\left[16\right.$, Sect 6.6]; the structure of the connection $\frac{D}{d s}$ resp of (2.5) then encodes the tangent cross ratio function: for arbitrary lifts $\xi$ and $\hat{\xi}$ of the curves of a Ribaucour pair (2.5) reads

$$
\hat{\xi}^{\prime}=2 c r \frac{(\xi, \hat{\xi})}{\left(\xi^{\prime}, \xi^{\prime}\right)} \xi^{\prime} \bmod \langle\xi, \hat{\xi}\rangle .
$$

As $\frac{D}{d s}$ is a metric connection, every initial point $\left\langle\hat{\xi}\left(s_{0}\right)\right\rangle \in S^{n}=\mathbb{P}\left(\mathcal{L}^{n+1}\right)$ yields a unique Ribaucour transform of a given curve $\langle\xi\rangle: I \rightarrow S^{n}$ via a $\frac{D}{d s}$-parallel section $\hat{\xi}: I \rightarrow \mathcal{L}^{n+1}$. A similar construction of Ribaucour transforms via parallel sections of a connection is possible in higher dimensions, though further conditions on the tangent cross ratios in curvature directions and initial point need to be satisfied.

Clearly, the tangent cross ratio of a Ribaucour pair $(x, \hat{x})$ of curves is parameter dependent. Considering differentials instead of derivatives we obtain the infinitesimal cross ratio

$$
x^{\prime} d s(\hat{x}-x)^{-1} \hat{x}^{\prime} d s(\hat{x}-x)^{-1}=c r d s^{2},
$$

a parameter independent quadratic differential. This resonates well with the Darboux transformation of an isothermic surface preserving a holomorphic quadratic differential (polarization) that occurs from a factorization of its Hopf differential, see [16, Lemma 5.2.12, Sect 5.4.14] ] and [7, Lemma 2.1, Thm 2.7]. Thus to introduce the Darboux transformation for curves we will consider a (non-vanishing) reference quadratic differential (polarization) $\frac{d s^{2}}{m}$ as part of the data.

Definition 2.4 A Ribaucour pair of curves $x, \hat{x}:\left(I, \frac{d s^{2}}{m}\right) \rightarrow \mathbb{R}^{n}$ on a polarized domain $\left(I, \frac{d s^{2}}{m}\right), m: I \rightarrow \mathbb{R}^{\times}$, will be called a Darboux pair if its infinitesimal cross ratio is a constant multiple of the reference polarization,

$$
x^{\prime}(\hat{x}-x)^{-1} \hat{x}^{\prime}(\hat{x}-x)^{-1}=\frac{\mu}{m} \text { with } \mu \equiv \text { const } \in \mathbb{R} .
$$

Either curve of a Darboux pair is a Darboux transform of the other polarized curve.

Clearly, since the tangent cross ratio of a Ribaucour pair $(x, \hat{x})$ is symmetric in $x$ and $\hat{x}$, so is the notion of Darboux transformation. An example of the Darboux transformation in Euclidean geometry is given by a tractrix construction, cf [18, Def 2.41]: if $y: I \rightarrow \mathbb{R}^{n}$ denotes an arc-length parametrized curve then

$$
x_{ \pm}:=y \pm \frac{1}{2 \sqrt{\mu}} y^{\prime}, \text { where } \mu \in(0, \infty),
$$


form a Darboux pair of curves with respect to their common arc-length polarization $\frac{d s^{2}}{m}=$ $\left|d x_{ \pm}\right|^{2}$; namely, with a principal normal field $n: I \rightarrow S^{n-1}$ of $y$ and the corresponding curvature $\kappa$ we obtain

$$
\left.\begin{array}{l}
x_{ \pm}^{\prime}=y^{\prime} \pm \frac{\kappa}{2 \sqrt{\mu}} n \\
x_{+}-x_{-}=\frac{1}{\sqrt{\mu}} y^{\prime}
\end{array}\right\} \Rightarrow c r d s^{2}=\mu\left|x_{ \pm}^{\prime} d s\right|^{2} .
$$

Given a polarized curve $x:\left(I, \frac{d s^{2}}{m}\right) \rightarrow \mathbb{R}^{n}$ every Darboux transform $\hat{x}:\left(I, \frac{d s^{2}}{m}\right) \rightarrow \mathbb{R}^{n}$ is obtained as a solution of a Riccati equation

$$
\hat{x}^{\prime}=\mu(\hat{x}-x)\left(m x^{\prime}\right)^{-1}(\hat{x}-x)
$$

as for the Ribaucour transformation the Darboux transformation is most naturally considered as a transformation of polarized curves in the conformal $n$-sphere $S^{n}=\mathbb{P}\left(\mathcal{L}^{n+1}\right)$, where the Riccati equation (2.9) linearizes and becomes Darboux's linear system, cf [15], that is, yields the zero curvature representation of the associated integrable system. In particular, for a polarized curve

$$
\langle\xi\rangle:\left(I, \frac{d s^{2}}{m}\right) \rightarrow S^{n}=\mathbb{P}\left(\mathcal{L}^{n+1}\right), \text { where } \xi: I \rightarrow \mathbb{R}^{n+1,1}
$$

denotes any light cone lift of the curve, we introduce a 1-parameter family of (flat) connections by

$$
\frac{D^{t}}{d s}:=\frac{d}{d s}-\frac{2 t}{m} \frac{\xi \wedge \xi^{\prime}}{\left(\xi^{\prime}, \xi^{\prime}\right)}
$$

Note that, as $\xi$ takes values in the light cone, the connections $\frac{D^{t}}{d s}$ do not depend on the choice of lift. Now Lemma 2.3 for the Ribaucour transformation directly yields a similar assertion for the Darboux transformation:

Definition and Corollary 2.5 The family $\left(\frac{D^{t}}{d s}\right)_{t \in \mathbb{R}}$ from (2.10) will be referred to as the isothermic family of connections of a polarized curve. The Darboux transforms of a polarized curve $\langle\xi\rangle:\left(I, \frac{d s^{2}}{m}\right) \rightarrow S^{n}$, with respect to a parameter $\mu \in \mathbb{R}$, are given by $\left.\frac{D^{t}}{d s}\right|_{t=\mu}$-parallel sections, that is, by solutions of Darboux's linear system

$$
\left.\frac{D^{t}}{d s}\right|_{t=\mu} \hat{\xi}=0
$$

Consequently, any polarized curve admits a $(1+n)$-parameter family of Darboux transforms as any choice of the spectral parameter $t=\mu$ and of an initial point in $S^{n}$ yields a unique Darboux transform via a $\left.\frac{D^{t}}{d s}\right|_{t=\mu}$-parallel light cone section. On the other hand, this new description (2.11) of the Darboux transformation lacks the symmetry of (2.7): thus our next goal is to understand how Darboux's linear system changes under the Darboux transformation.

Lemma 2.6 If $\langle\xi\rangle,\langle\hat{\xi}\rangle:\left(I, \frac{d s^{2}}{m}\right) \rightarrow S^{n}$ form a Darboux pair, then their isothermic loops of connections are related by a gauge transformation

$$
\frac{\hat{D}^{t}}{d s}=\Gamma_{\langle\xi\rangle}^{\langle\hat{\xi}\rangle}\left(1-\frac{t}{\mu}\right) \cdot \frac{D^{t}}{d s}, \text { where } \Gamma_{\langle\xi\rangle}^{\langle\hat{\xi}\rangle}(r) y:= \begin{cases}r y & \text { for } y \in\langle\hat{\xi}\rangle, \\ y & \text { for } y \in\langle\xi, \hat{\xi}\rangle^{\perp} \\ y / r & \text { for } y \in\langle\xi\rangle .\end{cases}
$$


Note that $\Gamma_{\langle\xi\rangle}^{\langle\hat{\xi}\rangle}(r) \in O\left(\mathbb{R}^{n+1,1}\right)$ realizes the Möbius geometric cross ratio of four points on a circle:

$$
\operatorname{cr}(\langle\hat{\xi}\rangle,\langle\eta\rangle,\langle\xi\rangle,\langle\zeta\rangle)=r \Leftrightarrow\langle\zeta\rangle=\left\langle\Gamma_{\langle\xi\rangle}^{\langle\hat{\xi}\rangle}(r) \eta\right\rangle
$$

namely, fixing relative normalizations of $\xi$ and $\hat{\xi}$ so that $(\xi, \hat{\xi})=-1$ and using $\xi \simeq o$ and $\hat{\xi} \simeq q=\infty$ as origin and point at infinity for Euclidean lifts (2.4) of $\langle\eta\rangle$ and $\langle\zeta\rangle$,

$$
\eta=\xi+y+\frac{1}{2}(y, y) \hat{\xi} \text { and } \zeta=\xi+z+\frac{1}{2}(z, z) \hat{\xi},
$$

we find, by computation of the (cyclic) Clifford algebra cross ratio in $\mathbb{R}^{n}$ of [14], that

$$
\operatorname{cr}(\langle\hat{\xi}\rangle,\langle\eta\rangle,\langle\xi\rangle,\langle\zeta\rangle)=y^{-1} z=r \Leftrightarrow \zeta=r \Gamma_{\langle\xi\rangle}^{\langle\hat{\xi}\rangle}(r) \eta .
$$

Changing viewpoint, (2.12) yields a parametrization of the circumcircle of three points, $\langle\xi\rangle,\langle\hat{\xi}\rangle,\langle\eta\rangle \in S^{n}$, by cross ratio:

$$
\mathbb{R} \cup\{\infty\} \ni r \mapsto\left\langle\Gamma_{\langle\xi\rangle}^{\langle\hat{\xi}\rangle}(r) \eta\right\rangle \in S^{n} .
$$

Lemma 2.6 follows directly from the following, more general statement, cf [9, Thm 3.10 and Prop 3.11]:

Lemma 2.7 Let $\langle\xi\rangle,\langle\hat{\xi}\rangle: I \rightarrow S^{n}$ be complementary, $\langle\xi(s)\rangle \neq\langle\hat{\xi}(s)\rangle$ for all $s \in I$, and $\gamma \in \Omega^{1}\left(\langle\xi\rangle \wedge\langle\xi\rangle^{\perp}\right)$ and $\hat{\gamma} \in \Omega^{1}\left(\langle\hat{\xi}\rangle \wedge\langle\hat{\xi}\rangle^{\perp}\right)$ 1-forms with values in $\langle\xi\rangle \wedge\langle\xi\rangle^{\perp}$ resp $\langle\hat{\xi}\rangle \wedge\langle\hat{\xi}\rangle^{\perp} \subset \mathfrak{o}\left(\mathbb{R}^{n+1,1}\right)$. Then

$$
(d+t \hat{\gamma})=\Gamma_{\langle\xi\rangle}^{\langle\hat{\xi}\rangle}\left(1-\frac{t}{\mu}\right) \cdot(d+t \gamma) \Leftrightarrow\left\{\begin{array}{l}
(d+\mu \gamma) \hat{\xi} \| \hat{\xi} \text { and } \\
(d+\mu \hat{\gamma}) \xi \| \xi .
\end{array}\right.
$$

We decompose $\mathbb{R}^{n+1,1}=\langle\xi\rangle \oplus\langle\hat{\xi}\rangle \oplus\langle\xi, \hat{\xi}\rangle^{\perp}$ and use the corresponding projections

$$
\pi: \mathbb{R}^{n+1,1} \rightarrow\langle\xi\rangle, \hat{\pi}: \mathbb{R}^{n+1,1} \rightarrow\langle\hat{\xi}\rangle \text { and } \varpi: \mathbb{R}^{n+1,1} \rightarrow\langle\xi, \hat{\xi}\rangle^{\perp},
$$

so that

$$
\mathrm{id}=\pi+\varpi+\hat{\pi} \text { and } \Gamma_{\langle\hat{\xi}\rangle}^{\langle\hat{\xi}\rangle}(r)=\frac{1}{r} \pi+\varpi+r \hat{\pi}
$$

note that, since $\xi$ and $\hat{\xi}$ are lightlike, for example $\pi(v)=\frac{(v, \hat{\xi})}{(\xi, \hat{\xi})} \xi$ for $v \in \mathbb{R}^{n+1,1}$. Thus we have

$$
\gamma=\pi \circ \gamma \circ \varpi+\varpi \circ \gamma \circ \hat{\pi} \text { and } \hat{\gamma}=\hat{\pi} \circ \gamma \circ \varpi+\varpi \circ \gamma \circ \pi
$$

as $\gamma \in \Omega^{1}\left(\langle\xi\rangle \wedge\langle\xi\rangle^{\perp}\right)$ yields:

- $\gamma \circ \pi=0$ since $\gamma(\xi)=0$;

- $\gamma \circ \varpi=\pi \circ \gamma \circ \varpi$ since $\gamma\left(\langle\xi\rangle^{\perp}\right) \subset\langle\xi\rangle$;

- $\gamma \circ \hat{\pi}=(\pi+\varpi) \circ \gamma \circ \hat{\pi}$ since $\gamma\left(\mathbb{R}^{n+1,1}\right) \subset\langle\xi\rangle^{\perp}$; further

- $\pi \circ \gamma \circ \hat{\pi}=0$ since $\gamma \in \Omega^{1}\left(\mathfrak{o}\left(\mathbb{R}^{n+1,1}\right)\right)$ is skew symmetric, so that $\gamma(\hat{\xi}) \perp \hat{\xi}$.

Similarly the derivative decomposes as

$d=D-\beta-\hat{\beta}, \quad$ where $\left\{\begin{array}{l}\beta:=-(\hat{\pi} \circ d \circ \varpi+\varpi \circ d \circ \pi) \in \Omega^{1}\left(\langle\hat{\xi}\rangle \wedge\langle\xi, \hat{\xi}\rangle^{\perp}\right) \\ \hat{\beta}:=-(\pi \circ d \circ \varpi+\varpi \circ d \circ \hat{\pi}) \in \Omega^{1}\left(\langle\xi\rangle \wedge\langle\xi, \hat{\xi}\rangle^{\perp}\right)\end{array}\right.$ and 
yield the derivatives of the maps $\langle\xi\rangle,\langle\hat{\xi}\rangle: I \rightarrow S^{n} \subset \mathbb{P}\left(\mathbb{R}^{n+1,1}\right)$, as $(\xi, d \xi)=0$ implies $\hat{\pi} \circ d \circ \pi=0$ so that $d \xi=-\beta \xi \bmod \xi$ and similarly for $\hat{\xi}$, and

$$
D=\pi \circ d \circ \pi+\hat{\pi} \circ d \circ \hat{\pi}+\varpi \circ d \circ \varpi
$$

is a metric connection on the vector bundle $\langle\xi\rangle \times\langle\hat{\xi}\rangle \times\langle\xi, \hat{\xi}\rangle^{\perp}$. Hence we compute

$$
\begin{aligned}
\Gamma_{\langle\xi\rangle}^{\langle\hat{\xi}\rangle}(r) \cdot(d+t \gamma)-(d+t \hat{\gamma}) & =\left(D-r \beta+\frac{1}{r}(t \gamma-\hat{\beta})\right)-(D+(t \hat{\gamma}-\beta)-\hat{\beta}) \\
& =\frac{1}{r}(t \gamma-(1-r) \hat{\beta})-(t \hat{\gamma}-(1-r) \beta) .
\end{aligned}
$$

Consequently, with $r=1-\frac{t}{\mu}$, we obtain the claimed result:

$$
\begin{gathered}
\Gamma_{\langle\xi\rangle}^{\langle\hat{\xi}\rangle}\left(1-\frac{t}{\mu}\right) \cdot(d+t \gamma)=(d+t \hat{\gamma}) \Leftrightarrow\left\{\begin{array}{l}
\mu \gamma=\hat{\beta} \\
\mu \hat{\gamma}=\beta
\end{array}\right\} \\
\Leftrightarrow\left\{\begin{array}{l}
(D-\beta+(\mu \gamma-\hat{\beta})) \hat{\xi}=0 \bmod \hat{\xi}, \\
(D+(\mu \hat{\gamma}-\beta)-\hat{\beta}) \xi=0 \bmod \xi .
\end{array}\right.
\end{gathered}
$$

Note that we imposed virtually no conditions on the maps $\langle\xi\rangle$ and $\langle\hat{\xi}\rangle$, in particular, we used no assumptions on the dimension of their common domain, nor on the 1-forms $\gamma$ and $\hat{\gamma}$, apart from the fact that they take values in the same places as the derivatives of $\langle\xi\rangle \operatorname{resp}\langle\hat{\xi}\rangle$ do. This reflects the utmost generality of the statement, cf [9].

It is now readily derived that the well known Bianchi permutability theorem for the Darboux transformation of isothermic surfaces descends to a permutability theorem for the Darboux transformation of polarized curves, cf [1, Sect 3] or [9, Sect 4.2]:

Theorem 2.8 Given Darboux transforms $\left\langle\xi_{0}\right\rangle,\left\langle\xi_{1}\right\rangle:\left(I, \frac{d s^{2}}{m}\right) \rightarrow S^{n}$ of a polarized curve $\langle\xi\rangle:\left(I, \frac{d s^{2}}{m}\right) \rightarrow S^{n}$ for different spectral parameters $\mu_{0} \neq \mu_{1}$, there is a simultaneous Darboux transform $\left\langle\xi_{01}\right\rangle$ of $\left\langle\xi_{i}\right\rangle(i=0,1)$ with parameters $\mu_{1-i}$, respectively, given algebraically by a constant cross ratio function

$$
\operatorname{cr}\left(\langle\xi\rangle,\left\langle\xi_{0}\right\rangle,\left\langle\xi_{01}\right\rangle,\left\langle\xi_{1}\right\rangle\right) \equiv \frac{\mu_{1}}{\mu_{0}} .
$$

By symmetry it is sufficient to show that $\left\langle\xi_{01}\right\rangle$ is a $\mu_{1}$-Darboux transform of $\left\langle\xi_{0}\right\rangle$, using a cross ratio identity, $\mathrm{cf}\left[16\right.$, Sect 4.9.11]. Thus we determine $\left\langle\xi_{01}\right\rangle$ from

$$
\operatorname{cr}\left(\left\langle\xi_{0}\right\rangle,\left\langle\xi_{1}\right\rangle,\langle\xi\rangle,\left\langle\xi_{01}\right\rangle\right)=1-\frac{\mu_{1}}{\mu_{0}}, \text { hence }\left\langle\xi_{01}\right\rangle=\left\langle\Gamma_{\langle\xi\rangle}^{\left\langle\xi_{0}\right\rangle}\left(1-\frac{\mu_{1}}{\mu_{0}}\right) \xi_{1}\right\rangle
$$

by (2.12); assuming that $\xi_{0}$ is a $\left.\frac{D^{t}}{d s}\right|_{t=\mu_{0}}$-parallel section, $\left.\frac{D^{t}}{d s}\right|_{t=\mu_{0}} \xi_{0}=0$, and using Lemma 2.6 we obtain

$$
\begin{aligned}
\left.\left(\frac{D^{t}}{d s}\right)_{0}\right|_{t=\mu_{1}} \xi_{01} & =\left(\left.\Gamma_{\langle\xi\rangle}^{\left\langle\xi_{0}\right\rangle}\left(1-\frac{\mu_{1}}{\mu_{0}}\right) \cdot \frac{D^{t}}{d s}\right|_{t=\mu_{1}}\right)\left(\Gamma_{\langle\xi\rangle}^{\left\langle\xi_{0}\right\rangle}\left(1-\frac{\mu_{1}}{\mu_{0}}\right) \xi_{1}\right) \\
& =\Gamma_{\langle\xi\rangle}^{\left\langle\xi_{0}\right\rangle}\left(1-\frac{\mu_{1}}{\mu_{0}}\right)\left(\left.\frac{D^{t}}{d s}\right|_{t=\mu_{1}} \xi_{1}\right)=0 .
\end{aligned}
$$

The relation between the isothermic loops of connections in a "Bianchi quadrilateral" is now obtained from Lemma 2.6; in fact, a more abstract statement holds true, that yields the key to an analogous approach to discrete isothermic nets, cf [11, Lemma 2.6] or [9, Lemma 4.7]: 


$$
\begin{aligned}
\Gamma_{\left\langle\xi_{0}\right\rangle}^{\left\langle\xi_{01}\right\rangle}\left(1-\frac{t}{\mu_{1}}\right) \circ \Gamma_{\langle\xi\rangle}^{\left\langle\xi_{0}\right\rangle}\left(1-\frac{t}{\mu_{0}}\right) & =\Gamma_{\left\langle\xi_{0}\right\rangle}^{\left\langle\xi_{1}\right\rangle}\left(\frac{1-t / \mu_{1}}{1-t / \mu_{0}}\right) \\
& =\Gamma_{\left\langle\xi_{1}\right\rangle}^{\left\langle\xi_{01}\right\rangle}\left(1-\frac{t}{\mu_{0}}\right) \circ \Gamma_{\langle\xi\rangle}^{\left\langle\xi_{1}\right\rangle}\left(1-\frac{t}{\mu_{1}}\right) .
\end{aligned}
$$

As for isothermic surfaces, $\operatorname{cf}$ [16, Sect 5.6.8], or isothermic submanifolds, cf [9, Sect 4.3], the "Bianchi cube" permutability theorem for the Darboux transformation of polarized curves is now obtained in a completely algebraic way from Theorem 2.8 using (2.13) or the hexahedron lemma of [16, Sect 4.9.13], that is, the "3D consistency of discrete isothermic nets", see [5, Thm 4.26]:

Theorem 2.9 Given three Darboux transforms $\left\langle\xi_{i}\right\rangle, i=0,1,2$, of a polarized curve $\langle\xi\rangle$ : $\left(I, \frac{d s^{2}}{m}\right) \rightarrow S^{n}$ with different parameters, $\mu_{i} \neq \mu_{j}$ for $i \neq j$, there is a simultaneous Darboux transform $\left\langle\xi_{012}\right\rangle$, with parameters $\mu_{k}$, of the simultaneous Darboux transforms $\left\langle\xi_{i j}\right\rangle$ of $\left\langle\xi_{i}\right\rangle$ and $\left\langle\xi_{j}\right\rangle$, where $i, j, k \in\{0,1,2\}$ are pairwise distinct.

\section{Semi-discrete isothermic surfaces}

We are now prepared to define semi-discrete isothermic surfaces, as sequences of Darboux transforms of curves-where we will parametrize the sequence by a graph $G=(V, E)$ and oriented edges $(i j) \in E$ will be denoted by the ordered pair of their endpoints $i, j \in V$.

Definition 3.1 A map $\langle\xi\rangle: \Sigma \rightarrow S^{n},(i, s) \mapsto\left\langle\xi_{i}(s)\right\rangle$, on a semi-discrete domain $\Sigma=G \times I$ will be called a semi-discrete isothermic surface if there is a polarization $\frac{d s^{2}}{m}$ on $I$ so that any adjacent curves $\left\langle\xi_{i}\right\rangle$ and $\left\langle\xi_{j}\right\rangle,(i j) \in E$, form a Darboux pair of curves on the polarized interval $\left(I, \frac{d s^{2}}{m}\right)$.

Apart from a sign issue this definition recovers the semi-discrete isothermic surfaces of [19]. With a stereographic projection

$$
x: \Sigma \rightarrow \mathbb{R}^{n},\langle\xi\rangle=\left\langle o+x+\frac{1}{2}(x, x) q\right\rangle,
$$

of $\langle\xi\rangle$ the condition of adjacent curves forming Darboux pairs can be formulated using the cross ratio condition (2.7),

$$
x_{i}^{\prime}\left(x_{j}-x_{i}\right)^{-1} x_{j}^{\prime}\left(x_{j}-x_{i}\right)^{-1}=\frac{\mu_{i j}}{m} .
$$

Then, reality of the cross ratio is equivalent to two adjacent curves enveloping a circle congruence while squaring the (real) cross ratio yields

$$
\mu_{i j}^{2}=\frac{m^{2}\left(x_{i}^{\prime}, x_{i}^{\prime}\right)\left(x_{j}^{\prime}, x_{j}^{\prime}\right)}{\left(x_{j}-x_{i}\right)^{4}}=\left(\frac{v_{i} v_{j}}{\left(x_{j}-x_{i}, x_{j}-x_{i}\right)}\right)^{2}, \text { where } v:=\sqrt{m\left(x^{\prime}, x^{\prime}\right)} .
$$

Note that we need to assume $m>0$ here; $\mu_{i j}<0$ then yields the semi-discrete isothermic surfaces of [19, Cor 4.2], $\operatorname{cf}\left[19\right.$, Lemma 3.3] with $\sigma=-\frac{1}{\mu}$ and $\tau=\frac{1}{m}$. Below we drop this restriction on the sign of the tangent cross ratio of adjacent curves and also allow Darboux transformations such as the ones from (2.8), or those of the generators of a cylinder to the corresponding curvature lines of a bubbleton, $\operatorname{cf}[17$, Sect 9]. 
As in the smooth and fully discrete cases, cf [5, Thms 1.32 and 4.31], a semi-discrete isothermic surface can be characterized by the existence of a Moutard lift

$$
\xi: \Sigma \rightarrow \mathcal{L}^{n+1}, \text { where } A(\xi, \xi)_{i j}:=\xi_{i j}^{\prime} \wedge d_{i j} \xi=0 \text { with }\left\{\begin{array}{l}
d_{i j} \xi:=\xi_{j}-\xi_{i}, \\
\xi_{i j}:=\frac{1}{2}\left(\xi_{i}+\xi_{j}\right)
\end{array}\right.
$$

denoting the discrete derivative resp edge function of $\xi$, that is, the existence of a light cone lift $\xi$ with vanishing (algebraic) area element $A(\xi, \xi) d s=0$. Namely, if $\xi$ satisfies (3.2) then, by (2.6),

$$
\xi_{i j}^{\prime} \| d_{i j} \xi=0 \bmod \left\langle\xi_{i}, \xi_{j}\right\rangle \Rightarrow c r=-\frac{\left(\xi_{i}^{\prime}, \xi_{i}^{\prime}\right)}{2\left(\xi_{i}, \xi_{j}\right)}=-\frac{\left(\xi_{j}^{\prime}, \xi_{j}^{\prime}\right)}{2\left(\xi_{i}, \xi_{j}\right)},
$$

in particular, $d_{i j}\left(\xi^{\prime}, \xi^{\prime}\right)=0$; further $\left(\xi_{i}, \xi_{j}\right)^{\prime}=2\left(\xi_{i j}, \xi_{i j}\right)^{\prime}=4\left(\xi_{i j}, \xi_{i j}^{\prime}\right)=0$, as $\xi_{i j}^{\prime} \| d_{i j} \xi$ again, showing that $\langle\xi\rangle$ is isothermic with

$$
c r=\frac{\mu}{m}, \quad \text { where } \begin{cases}m:=\frac{1}{\left(\xi^{\prime}, \xi^{\prime}\right)} & \text { so that } d_{i j} m=0 \\ \mu_{i j}:=\frac{1}{\left(d_{i j} \xi, d_{i j} \xi\right)} & \text { so that } \mu_{i j}^{\prime}=0\end{cases}
$$

Conversely, if $\langle x\rangle$ is isothermic with $c r=\frac{\mu}{m}$, where $m>0$ without loss of generality, then we obtain a Moutard lift by

$$
\xi:= \pm \frac{x}{\sqrt{m\left(x^{\prime}, x^{\prime}\right)}}, \text { where } \mu_{i j}\left(\xi_{i}, \xi_{j}\right)<0
$$

fixes the sign of $\xi$ up to a global sign choice-clearly $m\left(\xi^{\prime}, \xi^{\prime}\right) \equiv 1$, hence using (2.6) twice we learn that $\xi_{j}^{\prime}=2 \mu\left(\xi_{i}, \xi_{j}\right) \xi_{i}^{\prime}=4 \mu^{2}\left(\xi_{i}, \xi_{j}\right)^{2} \xi_{j}^{\prime} \bmod \left\langle\xi_{i}, \xi_{j}\right\rangle$ and, in particular, $\left(\xi_{i}, \xi_{j}\right)=-\frac{1}{2 \mu_{i j}} ;$ consequently,

$$
\xi_{i j}^{\prime}=0 \bmod \left\langle\xi_{i}, \xi_{j}\right\rangle \text { and } \xi_{i j}^{\prime} \perp \xi_{i j} \text { hence } \xi_{i j}^{\prime} \| d_{i j} \xi .
$$

Our definition has the full isothermic transformation theory built in: clearly, it directly lends itself to define the Darboux transformation of semi-discrete isothermic surfaces via the Darboux transformation of polarized curves and the Bianchi permutability theorem Theorem 2.8, showing that the Darboux transformations of curves "fit together" to form a new semi-discrete isothermic surface. The Bianchi cube Theorem 2.9 then yields the semi-discrete analogue of the usual Bianchi permutability theorem Theorem 2.8, cf [1].

Note that the curves of the original semi-discrete isothermic surface and of its Darboux transforms become indistinguishable or, otherwise said, a Darboux transform leads to a single "larger" semi-discrete isothermic surface with

$$
\tilde{\Sigma}=\tilde{G} \times I, \quad \tilde{V}=V \dot{\cup} V \text { and } \tilde{E}=E \dot{\cup} V \dot{\cup} E
$$

as a domain, that is, with two copies of the original graph $G$ and "vertical" edges added between corresponding points of the two copies.

Similarly, a "Christoffel duality" for polarized curves in $\mathbb{R}^{n}$, together with a suitable permutability theorem, yields a Christoffel duality for semi-discrete isothermic surfaces, $\mathrm{cf}$ [19, Thm 4.3], as long as there are no cycles in the discrete part $G$ of the domain $\Sigma$ of the semi-discrete isothermic surface.

Definition 3.2 Two curves $x, x^{*}:\left(I, \frac{d s^{2}}{m}\right) \rightarrow \mathbb{R}^{n}$ on a polarized domain will be said to be Christoffel dual if

$$
d x d x^{*}=x^{\prime}\left(x^{*}\right)^{\prime} d s^{2}=\frac{d s^{2}}{m}
$$


This duality for polarized curves yields the first equation of $[19,(10)]$, the second yields permutability with the Darboux transformation:

Theorem 3.3 Let $x:\left(I, \frac{d s^{2}}{m}\right) \rightarrow \mathbb{R}^{n}$ be a polarized curve with Christoffel dual $x^{*}$ and a Darboux transform $\hat{x}$, that is, $\hat{x}^{\prime}=\mu(\hat{x}-x)\left(x^{*}\right)^{\prime}(\hat{x}-x)$; then

$$
\hat{x}^{*}:=x^{*}+\frac{1}{\mu(\hat{x}-x)}
$$

is simultaneously Christoffel dual to $\hat{x}$ and a Darboux transform of $x^{*}$.

It is straightforward to verify the claim, using that Christoffel duality is involutive and by computing

$$
\left(m \hat{x}^{\prime}\right)^{-1}=\left(\hat{x}^{*}\right)^{\prime}=\mu\left(\hat{x}^{*}-x^{*}\right) x^{\prime}\left(\hat{x}^{*}-x^{*}\right) .
$$

This permutability theorem yields a version of the consistency check in [19] — thus it shows that Christoffel duals of consecutive curves of a semi-discrete isothermic surface can be positioned to form a new semi-discrete isothermic surface, its Christoffel dual, as long as the original surface has no discrete cycles. In fact, as long as $m>0>\mu$ our Christoffel duality is the same as the one of [19], as the defining equations coincide:

$$
\left(x^{*}\right)^{\prime}=\frac{1}{m x^{\prime}} \text { and } d_{i j} x^{*}=\frac{1}{\mu_{i j} d_{i j} x} .
$$

As in the smooth and fully discrete cases, cf [16, Sect 5.3.12] resp [2, Sect 7], this Christoffel transformation gives rise to a "Weierstrass representation" for semi-discrete minimal surfaces, cf [19, Sect 5] and [22].

To obtain the Calapso transformation, or "conformal deformation", of a polarized curve we introduce a (family of) gauge transformation(s) that trivialize the (flat) connections of its isothermic family of connections (2.10):

Definition 3.4 Let $\langle\xi\rangle:\left(I, \frac{d s^{2}}{m}\right) \rightarrow S^{n}$ be a polarized curve with isothermic family of connections $\frac{D^{t}}{d s}$;

$$
T^{t}:\left(I, \frac{d s^{2}}{m}\right) \rightarrow O\left(\mathbb{R}^{n+1,1}\right) \text { with } \frac{d}{d s} \circ T^{t}=T^{t} \circ \frac{D^{t}}{d s} \text { for } t \in \mathbb{R}^{\times}
$$

are called the Calapso transformations of $\langle\xi\rangle$, each curve $\left\langle\xi^{t}\right\rangle:=\left\langle T^{t} \xi\right\rangle$ is a Calapso transform of $\langle\xi\rangle$.

Note that the Calapso transformations $T^{t}$ can be chosen to take values in the orthogonal group since the connections $\frac{D^{t}}{d s}$ are metric connections; then they are unique up to post-composition by a (lift of a) Möbius transformation.

To carry this Calapso deformation for curves over to one for semi-discrete isothermic surfaces we will again require a permutability theorem; to this end, we will need to gain control of the Calapso transformations of the Calapso and Darboux transforms of a polarized curve.

Thus let $\langle\tilde{\xi}\rangle$ denote a Calapso transform of a polarized curve $\langle\xi\rangle$, that is, $\tilde{\xi}=T^{\tau} \xi$ for some $\tau \in \mathbb{R}$. Then (3.4) yields $\tilde{\xi}^{\prime}=T^{\tau} \xi^{\prime}$, hence

$$
\frac{\tilde{D}^{t}}{d s} \circ T^{\tau}=\left(\frac{d}{d s}-\frac{2 t}{m} \frac{\tilde{\xi} \wedge \tilde{\xi}^{\prime}}{\left(\tilde{\xi}^{\prime}, \tilde{\xi}^{\prime}\right)}\right) \circ T^{\tau}=T^{\tau} \circ\left(\frac{D^{\tau}}{d s}-\frac{2 t}{m} \frac{\xi \wedge \xi^{\prime}}{\left(\xi^{\prime}, \xi^{\prime}\right)}\right)=T^{\tau} \circ \frac{D^{\tau+t}}{d s}
$$


so that

$$
\tilde{T}^{t} T^{\tau} \circ{\frac{D^{\tau+t}}{d s}}^{\tau}=\tilde{T}^{t} \circ \frac{\tilde{D}^{t}}{d s} \circ T^{\tau}=\frac{d}{d s} \circ \tilde{T}^{t} T^{\tau} \Rightarrow \tilde{T}^{t} T^{\tau}=T^{\tau+t} .
$$

For a Darboux transformation $\langle\hat{\xi}\rangle$ of $\langle\xi\rangle$, where $\hat{\xi}$ satisfies (2.11), $\left.\frac{D^{t}}{d s}\right|_{t=\mu} \hat{\xi}=0$, we apply Lemma 2.6 to obtain

$$
\begin{aligned}
& \hat{T}^{t} \Gamma_{\langle\xi\rangle}^{\langle\hat{\xi}\rangle}\left(1-\frac{t}{\mu}\right) \circ \frac{D^{t}}{d s}=\hat{T}^{t} \circ \frac{\hat{D}^{t}}{d s} \circ \Gamma_{\langle\xi\rangle}^{\langle\hat{\xi}\rangle}\left(1-\frac{t}{\mu}\right) \\
& \quad=\frac{d}{d s} \circ \hat{T}^{t} \Gamma_{\langle\xi\rangle}^{\langle\hat{\xi}\rangle}\left(1-\frac{t}{\mu}\right) \Rightarrow \hat{T}^{t} \Gamma_{\langle\xi\rangle}^{\langle\hat{\xi}\rangle}\left(1-\frac{t}{\mu}\right)=T^{t} .
\end{aligned}
$$

Thus we have proved the following:

Lemma 3.5 Up to post-composition by a Möbius transformation:

(i) $\tilde{T}^{t} T^{\tau}=T^{\tau+t}$ for a Calapso transform $\langle\tilde{\xi}\rangle=\left\langle T^{\tau} \xi\right\rangle$ of $\langle\xi\rangle$;

(ii) $\hat{T}^{t} \Gamma_{\langle\xi\rangle}^{\langle\hat{\xi}\rangle}\left(1-\frac{t}{\mu}\right)=T^{t}$ for a Darboux transform $\langle\hat{\xi}\rangle$ of $\langle\xi\rangle$, where $\left.\frac{D^{t}}{d s}\right|_{t=\mu} \hat{\xi}=0$.

In particular, if $\langle\hat{\xi}\rangle$ is a Darboux transform of $\langle\xi\rangle$ with parameter $\mu$, that is, $\left.\frac{D^{t}}{d s}\right|_{t=\mu} \hat{\xi}=0$, then we learn from Lemma 3.5 (ii) that

$$
\left\langle\hat{T}^{\tau} \hat{\xi}\right\rangle=\left\langle T^{\tau} \hat{\xi}\right\rangle \text { for } \tau \neq \mu
$$

hence, by Lemma 3.5 (i),

$$
\left\langle\tilde{T}^{\mu-\tau} \hat{T}^{\tau} \hat{\xi}\right\rangle=\left\langle\tilde{T}^{\mu-\tau} T^{\tau} \hat{\xi}\right\rangle=\left\langle T^{\mu} \hat{\xi}\right\rangle \equiv \text { const },
$$

showing that $\left\langle T^{\tau} \hat{\xi}\right\rangle$ yields simultaneously a Calapso transform of $\langle\hat{\xi}\rangle$ and a Darboux transform of $\left\langle T^{\tau} \xi\right\rangle$. Thus we obtain the desired permutability theorem for the Darboux and Calapso transformations, which ensures that the Calapso transformation for curves extends to one for semi-discrete isothermic surfaces, as long as the surfaces do not have discrete cycles.

Theorem 3.6 Suppose that $\langle\xi\rangle,\langle\hat{\xi}\rangle:\left(I, \frac{d s^{2}}{m}\right) \rightarrow S^{n}$ form a Darboux pair with parameter $\mu \in \mathbb{R}^{\times}$, and let $\tau \neq \mu$. Then their Calapso transforms $\left\langle T^{\tau} \xi\right\rangle$ and $\left\langle\hat{T}^{\tau} \hat{\xi}\right\rangle$ form, if suitably positioned, a Darboux pair with parameter $\mu-\tau$.

Thus we have learned that the transformations of curves extend to sequences of Darboux transforms of curves, that is, to semi-discrete isothermic surfaces, by means of suitable permutability theorems:

Theorem and Definition 3.7 The Darboux, Christoffel and Calapso transformations for polarized curves extend to corresponding transformations of cycle-free semi-discrete isothermic surfaces.

An alternative approach to the transformations of semi-discrete isothermic surfaces is more directly based on a family of semi-discrete flat connections, similar to those of Definition and Corollary 2.5:

Definition and Theorem 3.8 A semi-discrete connection on a vector bundle $X$ over a domain $\Sigma=G \times I, G=(V, E)$, is a pair $(\Gamma, \nabla)$, consisting of connections over each smooth component, $\nabla_{i}$ on $X_{i}$ for each $i \in V$, and vector bundle isomorphisms between 
components, $\Gamma_{i j}: X_{j} \rightarrow X_{i}$ for each $(i j) \in E$; a semi-discrete connection is flat if all $\nabla_{i}$ are flat and are gauge equivalent via $\Gamma_{i j}$,

$$
\forall i \in V: R^{\nabla_{i}}=0 \text { and } \forall(i j) \in E: \Gamma_{i j} \cdot \nabla_{j}=\nabla_{i} .
$$

A semi-discrete surface $\langle\xi\rangle: \Sigma \rightarrow S^{n}$ is isothermic if and only if there are functions $m$ and $\mu$ on $I$ and $E$, respectively, so that the associated isothermic loop of connections $\left(\Gamma^{t}, \nabla^{t}\right)_{t \in \mathbb{R}}$ consists of flat connections, where

$$
\Gamma_{i j}^{t}:=\Gamma_{\left\langle\xi_{j}\right\rangle}^{\left\langle\xi_{i}\right\rangle}\left(1-\frac{t}{\mu_{i j}}\right) \text { and } \nabla_{i}^{t}:=\left.\frac{D^{t}}{d s}\right|_{i}=\frac{d}{d s}-\frac{2 t}{m} \frac{\xi_{i} \wedge \xi_{i}^{\prime}}{\left(\xi_{i}^{\prime}, \xi_{i}^{\prime}\right)} .
$$

This characterization of semi-discrete isothermic surfaces is an immediate consequence of Definition 3.1 in conjunction with Lemma 2.7. To obtain the Darboux and Calapso transformations only the flatness assertion is required, which already follows from the simpler statement of Lemma 2.6: then the following characterizations of the Darboux and Calapso transformations follow directly from their definitions and the corresponding characterizations Definition and Corollary 2.5 and Definition 3.4 for polarized curves, cf Lemma 3.5.

Theorem 3.9 Let $\langle\xi\rangle: \Sigma \rightarrow S^{n}$ be semi-discrete isothermic with isothermic loop of connections $\left(\Gamma^{t}, \nabla^{t}\right)_{t \in \mathbb{R}}$. Then

(i) the Darboux transforms $\langle\hat{\xi}\rangle$ of $\langle\xi\rangle$, with respect to a parameter $\mu$, are given by $\left(\Gamma^{\mu}, \nabla^{\mu}\right)$ parallel sections, that is, by solutions of Darboux's linear system

$$
\forall(i j) \in E: \Gamma_{i j}^{\mu} \hat{\xi}_{j}=\hat{\xi}_{i} \text { and } \forall i \in V: \nabla_{i}^{\mu} \hat{\xi}_{i}=0 ;
$$

(ii) the Calapso transforms $\left\langle\xi^{t}\right\rangle$ of $\langle\xi\rangle$ are given as images of $\langle\xi\rangle$ under trivializing gauge transformations,

$$
\left\langle\xi^{t}\right\rangle=\left\langle T^{t} \xi\right\rangle, \text { where } T^{t} \cdot\left(\Gamma^{t}, \nabla^{t}\right)=\left(\mathrm{id}, \frac{d}{d s}\right) \Leftrightarrow\left\{\begin{array}{l}
\forall(i j) \in E: T_{i}^{t} \circ \Gamma_{i j}^{t}=T_{j}^{t}, \\
\forall i \in V: T_{i}^{t} \circ \nabla_{i}^{t}=\frac{d}{d s} \circ T_{i}^{t} .
\end{array}\right.
$$

At this point the full integrable theory of isothermic surfaces is available and corresponding results in the semi-discrete setting can be obtained in a completely analogous way as in the smooth or fully discrete settings. For example, we may now define special isothermic surfaces as those semi-discrete isothermic surfaces that admit a polynomial conserved quantity, i.e., a polynomial map

$$
p(t)=z t^{d}+y t^{d-1}+\cdots+q: \Sigma \rightarrow \mathbb{R}^{n+1,1} \text { with } \forall t \in \mathbb{R}:\left(\Gamma^{t}, \nabla^{t}\right) p(t)=0,
$$

cf [8, Defs 2.1 and 2.3] and [12, Defs 1 and 2]. Wheeling out the conserved quantity conditions

$$
(z t+q)_{i}=\Gamma_{i j}^{t}(z t+q)_{j} \text { and } 0=\frac{D^{t}}{d s}(z t+q)
$$

in the case of a linear conserved quantity $p(t)=z t+q$ yields $q \equiv$ const, $z \perp \xi$ and, cf $[12,(2.3)]$,

$$
d_{i j} z=\frac{1}{\mu_{i j}}\left(\pi_{i}-\pi_{j}\right) q \text { and } z^{\prime}=\frac{2}{m\left(\xi^{\prime}, \xi^{\prime}\right)}\left\{(q, \xi) \xi^{\prime}-\left(q, \xi^{\prime}\right) \xi\right\},
$$

where $\pi_{i}$ and $\pi_{j}$ denote the projections onto $\left\langle\xi_{i}\right\rangle$ resp $\left\langle\xi_{j}\right\rangle$, and $d_{i j} z=z_{j}-z_{i}$ the discrete derivative of $z$, as before; note that $\pi_{j}\left(d_{i j} z+\frac{1}{\mu_{i j}}\left(\pi_{j}-\pi_{i}\right) q\right)=\pi_{j}\left(z_{j}+\frac{1}{\mu_{i j}} q\right)$ since $z \perp \xi$. 
Thus normalizing $\xi=x$ so that $(x, q) \equiv-1$ we learn that $z$ is, up to scale, a Christoffel transform of $x$ in $\mathbb{R}^{n+1,1}$ :

$$
z^{\prime}=\frac{2}{m x^{\prime}} \text { and } d_{i j} z=\frac{2}{\mu_{i j} d_{i j} x} .
$$

As $\left(\Gamma^{t}, \nabla^{t}\right)$ are metric connections the (real) polynomial $|z t+q|^{2}$ has constant coefficients, in particular, we may without loss of generality assume $|z|^{2} \equiv 1$ as long as $|z| \neq 0$, that is, $p(t)=z t+q$ to be a normalized linear conserved quantity. Conversely, $p(t)=z t+q$ yields a (normalized) linear conserved quantity of $\langle\xi\rangle=\langle x\rangle$ as soon as

$$
z: \Sigma \rightarrow S^{3,1}:=\left\{y \in \mathbb{R}^{n+1,1} \mid(y, y)=1\right\} \text { with } H:=-(z, q)=\text { const }
$$

is (up to scale) a Christoffel transform of

$$
x: \Sigma \rightarrow Q^{3}=\left\{y \in \mathcal{L}^{n+1} \mid(y, q)=-1\right\} \subset \mathbb{R}^{n+1,1} .
$$

On the other hand, the two surfaces $x, z: \Sigma \rightarrow \mathbb{R}^{n+1,1}$ form a Christoffel pair if and only if their mixed area element

$$
A(x, z) d s:=\frac{1}{2}\left(x_{i j}^{\prime} \wedge d_{i j} z+z_{i j}^{\prime} \wedge d_{i j} x\right) d s \equiv 0,
$$

with $z_{i j}=\frac{z_{i}+z_{j}}{2}$, as before; cf [19, Sect 5] and [20, Sect 1.5], see also [5, Thm 4.42] or [6, Lemma 2.3].

Namely, suppose that $z$ and $x$ define parallel nets, that is, with suitable functions $a_{i}$ and $\alpha_{i j}$ we have

$$
z_{i}^{\prime}=a_{i} x_{i}^{\prime} \text { and } d_{i j} z=\alpha_{i j} d_{i j} x .
$$

For regularity we assume $d_{i j} x \not \mid x_{i}^{\prime}, x_{j}^{\prime}$ and that $x$ and $z$ are not homothetic. Integrability of $z$ then yields

$$
\left(d_{i j} z\right)^{\prime}=d_{i j}\left(z^{\prime}\right) \Leftrightarrow\left(\alpha_{i j}-a_{i}\right) x_{i}^{\prime}-\left(\alpha_{i j}-a_{j}\right) x_{j}^{\prime}=\alpha_{i j}^{\prime} d_{i j} x .
$$

In particular, we learn that $x_{i}^{\prime}, x_{j}^{\prime}$ and $d_{i j} x$ must be linearly dependent, that is, $x$ must be a conjugate net in $\mathbb{R}^{n+1,1}$, cf [21, Sect 2]; hence adjacent curves $x_{i}$ and $x_{j}$ form Ribaucour pairs, since $x$ maps into the conformal $n$-sphere, and $x$ is circular or a semi-discrete curvature line net, cf [19, Definition 1.1]. Now, we use (3.6) to analyze vanishing of the mixed area,

$$
A(x, z)_{i j}=0 \Leftrightarrow\left(\alpha_{i j}+a_{i}\right) x_{i}^{\prime}+\left(\alpha_{i j}+a_{j}\right) x_{j}^{\prime}=0 \bmod d_{i j} x \Leftrightarrow \alpha_{i j}^{2}=a_{i} a_{j} .
$$

We conclude that $a_{i} a_{j}>0$ and that there is a function $v: \Sigma \rightarrow \mathbb{R}^{\times}$with $a_{i}=\mp \frac{1}{v_{i}^{2}}$ and $\alpha_{i j}=\frac{1}{v_{i} v_{j}}$. Without loss of generality we may assume $a_{i}=-\frac{1}{v_{i}^{2}}$ to recover that vanishing of the mixed area is a characterization of the Koenigs duality of conjugate nets from [19, Definition 3.1], up to a sign:

$$
z_{i}^{\prime}=-\frac{1}{v_{i}^{2}} x_{i}^{\prime} \text { and } d_{i j} z=\frac{1}{v_{i} v_{j}} d_{i j} x .
$$

However, for a semi-discrete curvature line net, existence of a Koenigs dual implies isothermicity, and the Koenigs and Christoffel dualities coincide. To see this we use again integrability (3.6) of $z$, which now reads 


$$
\begin{aligned}
0 & =\left(\frac{1}{v_{i} v_{j}}\right)^{\prime} d_{i j} x+\left(\frac{1}{v_{i}}+\frac{1}{v_{j}}\right) d_{i j}\left(\frac{x^{\prime}}{v}\right) \\
& =\left(\frac{1}{v_{i} v_{j}}\right)^{\prime} d_{i j} x+\frac{2}{v_{i} v_{j}} d_{i j} x^{\prime}+\left(\frac{1}{v_{j}}-\frac{1}{v_{i}}\right)\left(\frac{x^{\prime}}{v}\right)_{i j} .
\end{aligned}
$$

Since $x$ is isotropic, $(x, x) \equiv 0$, we have $x_{i j} \perp d_{i j} x$, hence using $d_{i j}\left(\frac{x^{\prime}}{v}\right) \| d_{i j} x$ from (3.8) we obtain

$$
\begin{aligned}
\left(d_{i j} x,\left(\frac{x^{\prime}}{v}\right)_{i j}\right) & =d_{i j}\left(x, \frac{x^{\prime}}{v}\right)-\left(x_{i j}, d_{i j}\left(\frac{x^{\prime}}{v}\right)\right)=d_{i j}\left(x, \frac{x^{\prime}}{v}\right) \\
& =0 \Rightarrow d_{i j}\left(\frac{x^{\prime}}{v}\right) \perp\left(\frac{x^{\prime}}{v}\right)_{i j} ;
\end{aligned}
$$

further, taking inner product with $d_{i j} x$ in (3.8) the right term yields the second of the equations

$$
d_{i j}\left(\frac{1}{m}\right)=0 \text { and }\left(\frac{1}{\mu_{i j}}\right)^{\prime}=0, \text { where } \frac{1}{m}:=\frac{\left(x^{\prime}, x^{\prime}\right)}{v^{2}} \text { and } \frac{1}{\mu_{i j}}:=\frac{2\left(x_{i}, x_{j}\right)}{v_{i} v_{j}},
$$

cf (3.1): now (3.7) turns into Christoffel's equation (3.3) and (3.8) shows that $x_{j}$ is a $\mu_{i j}$ Darboux transform of $x_{i}$, that is, isothermicity of $x$, as

$$
\begin{aligned}
0 & =\left(\frac{1}{v_{i} v_{j}}\right)^{\prime} d_{i j} x+\left(\frac{1}{v_{i}}+\frac{1}{v_{j}}\right) d_{i j}\left(\frac{x^{\prime}}{v}\right) \\
& =\left(\frac{1}{v_{i}}+\frac{1}{v_{j}}\right)\left\{\left.\frac{1}{v_{j}} \frac{D^{t}}{d s}\right|_{t=\mu_{i j}} x_{j}-\frac{1}{v_{i}} \frac{\left(x_{i}^{\prime}, x_{j}\right)}{\left(x_{i}, x_{j}\right)} x_{j}\right\},
\end{aligned}
$$

where we use $\frac{\left(x_{i}^{\prime}, x_{j}\right)}{v_{i}}=\frac{\left(x_{i}, x_{j}^{\prime}\right)}{v_{j}}$ from $\left(\frac{x^{\prime}}{v}\right)_{i j} \perp d_{i j} x$ and $\frac{v_{i}^{\prime}}{v_{i}}+\frac{v_{j}^{\prime}}{v_{j}}=\frac{\left(x_{i}^{\prime}, x_{j}\right)+\left(x_{i}, x_{j}^{\prime}\right)}{\left(x_{i}, x_{j}\right)}$ from the definition of $\mu_{i j}$.

Note that these results descend to Euclidean ambient geometry; however, corresponding proofs in a purely Euclidean setting will require some arguments to be adapted, for example, by using the tangent cross ratio instead of isotropy of $x$ to obtain $m$ and $\mu$ as functions of one parameter.

Using the vanishing of the mixed area (3.5) we then arrive at characterizations of semidiscrete surfaces of constant mixed area mean curvature in space forms,

$$
\begin{aligned}
& H:=-\frac{A(x, n)}{A(x, x)}, \text { where } x: \Sigma \rightarrow Q^{3} \text { and } \\
& n: \Sigma \rightarrow P^{3}=\left\{y \in S^{3,1} \mid y \perp q\right\} \text { with } n \perp x
\end{aligned}
$$

defines a suitable tangent plane congruence for $x$ in $Q^{3}, \operatorname{cf}[19$, Prop 5.2] and [20, Definition 8] for the Euclidean case and see [10, Definition 2.3] for arbitrary ambient space form geometries in the fully discrete case: namely, consider

$$
z=n+H x \text { with } H=-(z, q)
$$

to see that $(x, n): \Sigma \rightarrow Q^{3} \times P^{3}$ has constant mean curvature if and only if $x$ has a suitable Christoffel dual $z: \Sigma \rightarrow S^{3,1}$, if and only if $\langle\xi\rangle=\langle x\rangle$ has a normalized linear conserved quantity $p(t)=z t+q$, cf [6, Lemma 4.1] and [10, Thm 2.8] resp [8, Prop 2.5] and [11, Sect 5]. 
Acknowledgments We would like to thank M Yasumoto for fruitful and enjoyable discussions around the subject. This work has been partially supported by the Austrian Science Fund (FWF) and the Japan Society for the Promotion of Science (JSPS) through the FWF/JSPS Joint Project Grant I1671-N26 "Transformations and Singularities".

Open Access This article is distributed under the terms of the Creative Commons Attribution 4.0 International License (http://creativecommons.org/licenses/by/4.0/), which permits unrestricted use, distribution, and reproduction in any medium, provided you give appropriate credit to the original author(s) and the source, provide a link to the Creative Commons license, and indicate if changes were made.

\section{References}

1. Bianchi, L.: Il teorema di permutabilità per le trasformazioni di Darboux delle superficie isoterme. Rend. Acc. Naz. Lincei. 13, 359-367 (1904)

2. Bobenko, A., Pinkall, U.: Discrete isothermic surfaces. J. Reine Angew. Math. 475, 187-208 (1996)

3. Bobenko, A., Hertrich-Jeromin, U.: Orthogonal nets and Clifford algebras. Tôhoku Math. Publ. 20, 7-22 (2001)

4. Bobenko, A., Suris, Y.: On organizing principles of discrete differential geometry. Geometry of spheres. Russ. Math. Surv. 62, 1-43 (2007)

5. Bobenko, A., Suris Y.: Discrete Differential Geometry. Integrable Structure. Graduate in Studies Mathematics, vol. 98. American Mathematical Society, Providence RI (2008)

6. Bobenko, A., Hertrich-Jeromin, U., Lukyanenko, I.: Discrete constant mean curvature nets in space forms: Steiner's formula and Christoffel duality. Discrete Comput. Geom. 52, 612-629 (2014)

7. Burstall, F.: Isothermic surfaces: conformal geometry, Clifford algebras and integrable systems. AMS Stud. Adv. Math. 36, 1-82 (2006)

8. Burstall, F., Santos, S.: Special isothermic surfaces of type $d$. J. Lond. Math. Soc. 85, 571-591 (2012)

9. Burstall, F., Donaldson, N., Pedit, F., Pinkall, U.: Isothermic submanifolds of symmetric $R$-spaces. J. Reine Angew. Math. 660, 191-243 (2011)

10. Burstall, F., Hertrich-Jeromin, U., Rossman W.: Discrete linear Weingarten surfaces. EPrint arXiv:math.DG/1406.1293 (2014)

11. Burstall, F., Hertrich-Jeromin, U., Rossman, W., Santos, S.: Discrete surfaces of constant mean curvature. RIMS Kokyuroku 1880, 133-179 (2014)

12. Burstall, F., Hertrich-Jeromin, U., Rossman, W., Santos, S.: Discrete special isothermic surfaces. Geom. Dedicata 174, 1-11 (2015)

13. Calini, A., Ivey, T.: Bäcklund transformations and knots of constant torsion. J. Knot Theory Ramif. 7, 719-746 (1998)

14. Cieśliński, J.: The cross ratio and Clifford algebras. Adv. Appl. Clifford Algebras 7, 133-139 (1997)

15. Darboux, G.: Sur les surfaces isothermiques. Ann. Sci. Éc. Norm. Sup. 16, 491-508 (1899)

16. Hertrich-Jeromin, U.: Introduction to Möbius Differential Geometry. London Mathematical Society Lecture Notes Series, vol. 300. Cambridge Univ Press, Cambridge (2003)

17. Hertrich-Jeromin, U., Pedit, F.: Remarks on the Darboux transform of isothermic surfaces. Doc. Math. 2 , 313-333 (1997)

18. Hoffmann T.: Discrete Differential Geometry of Curves and Surfaces. COE Lecture Notes 18, Kyushu Univ (2009)

19. Müller, C., Wallner, J.: Semi-discrete isothermic surfaces. Res. Math. 63, 1395-1407 (2013)

20. Müller, C.: Semi-discrete constant mean curvature surfaces. Math. Z. 279, 459-478 (2015)

21. Pottmann, H., Schiftner, A., Bo, P., Wallner, J.: Freeform surfaces from single curved panels. ACM Trans. Graph. 27, 76 (2008)

22. Rossman, W., Yasumoto, M.: Weierstrass representation for semi-discrete minimal surfaces, and comparison of various discretized catenoids. J. Math. Ind. 4, 109-118 (2012) 